\title{
Unveiling the Early Stages of the F-element Oxalate Growth Evolution with Cryo- TEM
}

Tenisha Meadows, Karen Kruska, Shalini Tripathi, Gabriel Hall, Jennifer Carter, Bruce McNamara, Christopher Armstrong, Jennifer Solits, Cyrena Parker and Edgar Buck

Pacific Northwest National Laboratory, Richland, Washington, United States

The oxalate anion has long been recognized as an excellent precipitating agent for trivalent lanthanides and actinides owing to the remarkably low solubility of f-element oxalates in acidic solutions [1]. Previous studies have shown morphologies of f-element oxalate are precipitated as agglomerates and preserved in the oxide phase following calcination [2]. There are indications that distinct morphologies arise depending on the solution formation conditions. This suggests it may be possible to predict the morphology of an $\mathrm{f}$ element oxide knowing the formation conditions or even deduce the formation conditions from the observed morphology in oxide agglomerates. Analysis of the particle nucleation and growth with in-situ electron microscopy has been performed with many other chemical systems and is an effective method for probing the particle formation dynamics [3,4]. One particular challenge is the rapid nucleation and growth of f-element oxalate precipitates. To capture the early stages of nucleation and growth of $\mathrm{f}$-element oxalates that result from the reaction of the f-metal nitrate and oxalic acid, we used cryo-transmission electron microscopy. A combination of optical and electron microscopies was used to investigate the full growth cycle.

$\mathrm{Nd}, \mathrm{U}$ and $\mathrm{Pu}$ nitrites were reacted with oxalic acid. The growth of oxalates was observed in-situ with both optical microscopy (OM) and scanning electron microscopy (SEM). The OM used was a Nikon 600MEL metallurgical microscope with a Linkham thermal stage and the SEM was a FEI (ThermoFisher Inc., Hilsboro, OR) Quanta 250FEG Environmental SEM. For the in-situ work, we deposited solutions into QuantomiX (El-Mul, Rehovot, Israel) QX102 wetSEM® cells. For cryo-TEM analysis the mixture was rapidly frozen in liquid ethane. Detailed characterization was performed using a JEOL (JEOL USA Inc., Peabody, MA) GrandARM ${ }^{\mathrm{TM}} 300 \mathrm{~F}$ probe corrected scanning transmission electron microscope (STEM) operated at $300 \mathrm{kV}$. The TEM was equipped with annular dark field (ADF) and bright-field (BF) detectors, and a Gatan Image Filter (Gatan Inc., Pleasanton, CA). Cryo-analysis was carried out in a Gatan liquid nitrogen cryo-transfer holder. All the equipment is part of the Radiochemical Microscopy Suite in the Radiochemical Processing Laboratory at PNNL.

The initial stages of the reaction are displayed in Figure 1. A 200mg/L solution of Nd(III) nitrate was reacted with oxalic acid. STEM-Electron Energy-Loss Spectroscopy (EELS) analysis reveals 50-100 nm crystallites connected by thin $\mathrm{C}$-rich nanowires. Further observation suggested that these nano-crystallites dissolve within a few minutes, and larger micron-sized metal oxalate particles form. Bulk scale x-ray diffraction indicated that this second phase may only be metastable in the solution. We postulate the precipitation of a third stable phase. In Figure 2A illustrates the process of $\mathrm{Pu}$ oxalate precipitation using energy dispersive spectroscopy (EDS) in an in-situ liquid cell experiment. The EDS spectrum of a Pu particle in solution is shown in Figure 2B. The count rate was kept low to prevent damage to the cell window. The precipitate size of $\sim 10 \mu \mathrm{m}$ after a few minutes demonstrates the need for cryo-observations which can arrest growth at well-defined intervals. Figure $2 \mathrm{C}$ shows a dried $\mathrm{Pu}$ oxalate from bulk scale precipitation. The $\mathrm{Pu}$ oxalate is characterized by elongated individual crystals that will aggregate to form various structures including rosettes and crosses. We will discuss the morphological changes in f-element oxalates that result from changes in the chemical precipitation conditions [5]. 

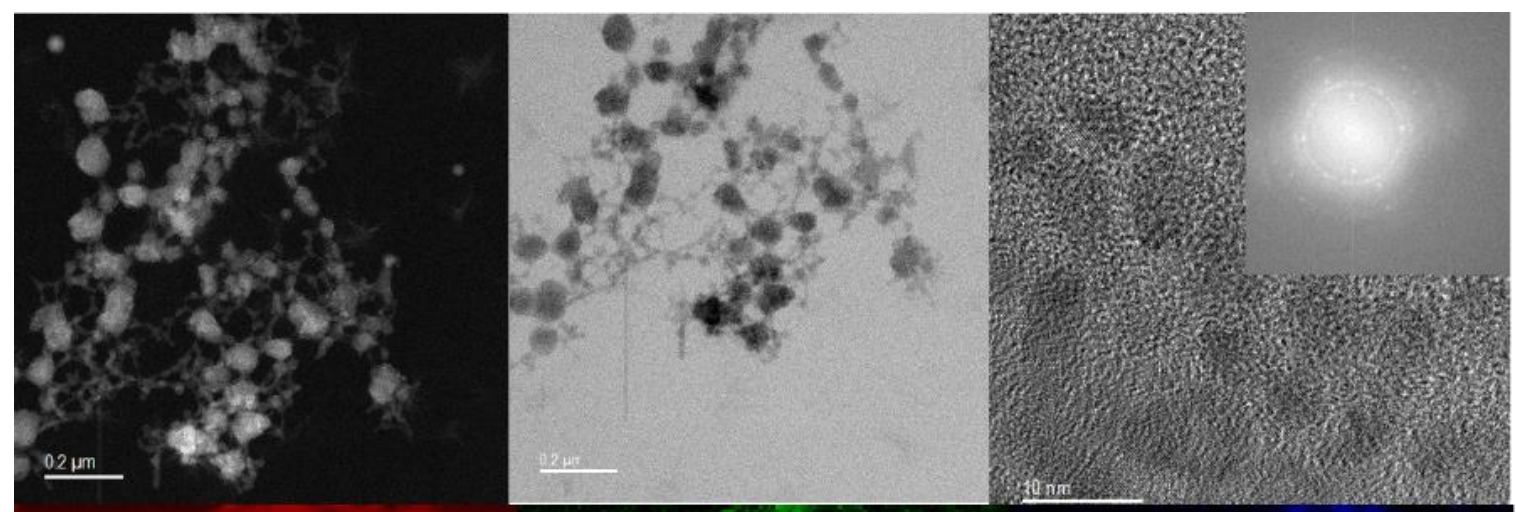

Carbon

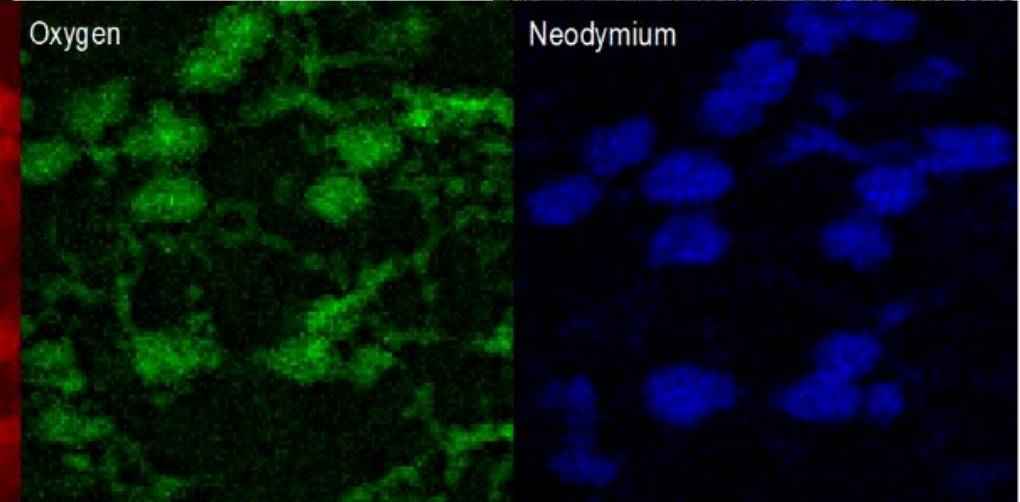

Figure 1. STEM- ADF and -BF images of the $\mathrm{Nd}$ (III) oxalate particles formed with $200 \mathrm{mg} \mathrm{Nd}$ in $1 \mathrm{M}$ HNO3 showing the formation of early crystallites in TEM mode. These particles that formed within the first few seconds of the reaction were only partially crystalline. STEM-EELS maps of carbon, oxygen, and neodymium.
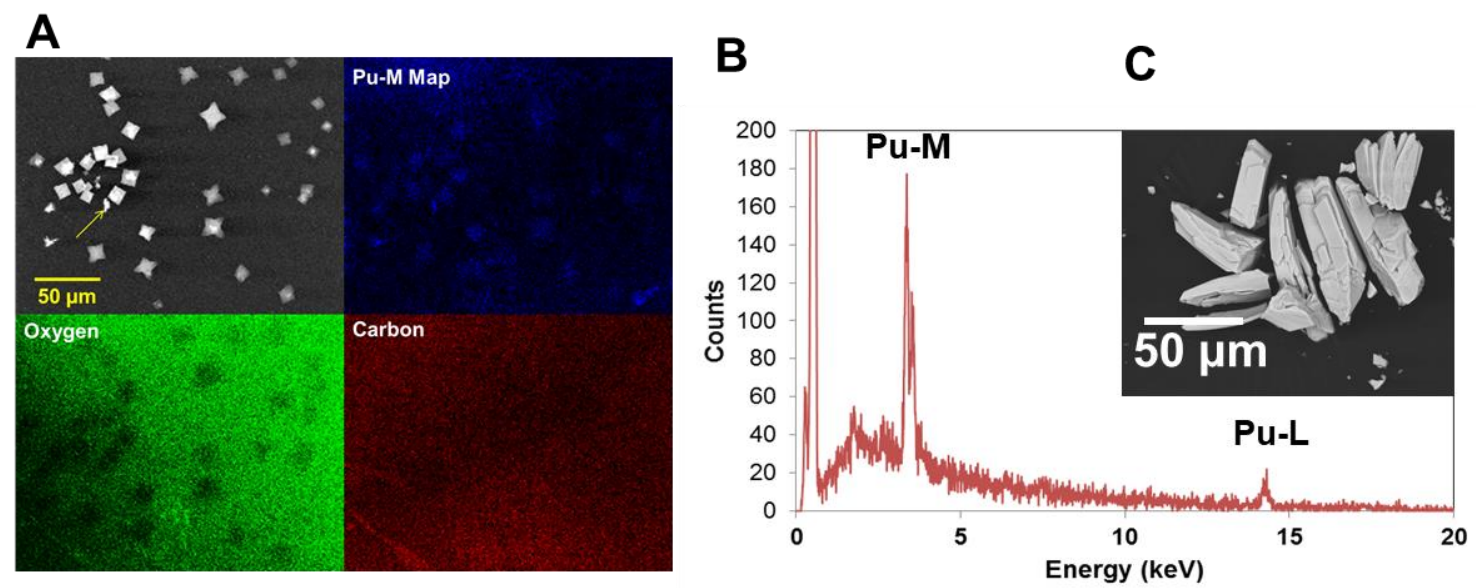

Figure 2. (A) In-situ liquid cell images and elemental SEM-EDS maps from Pu oxalate, (B) EDS from liquid cell showing the Pu M- and L- lines, (C) SEM of dried Pu oxalate. The morphology of the oxalates formed under slightly different conditions is striking.

References

[1] W. Runde, L. F. Brodnax, G. Goff, A. C. Bean, and B. L. Scott, "Directed Synthesis of Crystalline Plutonium(III) and (IV) Oxalates: Accessing Redox-Controlled Separations in Acidic Solutions," Inorganic Chemistry, vol. 48, no. 13, pp. 5967-5972, 2009/07/06 2009, doi: 10.1021/ic900344u. 
[2] V. Tyrpekl, M. Beliš, T. Wangle, J. Vleugels, and M. Verwerft, "Alterations of thorium oxalate morphology by changing elementary precipitation conditions," Journal of Nuclear Materials, vol. 493, pp. 255-263, 9// 2017, doi: https://doi.org/10.1016/j.jnucmat.2017.06.027.

[3] J. J. De Yoreo et al., "Crystallization by particle attachment in synthetic, biogenic, and geologic environments," Science, vol. 349, no. 6247, 2015-07-31 00:00:00 2015, doi: 10.1126/science.aaa6760.

[4] J. A. Soltis, W. C. Isley, M. Conroy, S. M. Kathmann, E. C. Buck, and G. J. Lumetta, "In situ microscopy across scales for the characterization of crystal growth mechanisms: the case of europium oxalate," CrystEngComm, 10.1039/C7CE01450C vol. 20, no. 20, pp. 2822-2833, 2018, doi: 10.1039/C7CE01450C.

[5] Work supported by the Department of Homeland Security. PNNL is operated for the U.S. Department of Energy (DOE) by Battelle Memorial Institute under Contract DE-AC06-76RL0 1830. 